\title{
Use of Ultra High Performance Liquid Chromatography-Tandem Mass Spectrometry to Demonstrate Decreased Serum Statin Levels after Extracorporeal LDL-Cholesterol Elimination
}

\author{
M. Bláha, ${ }^{1}$ H. Vlčková, ${ }^{2}$ L. Nováková, ${ }^{2}$ D. Solichová, ${ }^{3}$ P. Solich, ${ }^{2}$ M. Lánská, ${ }^{1}$ \\ J. Malý, ${ }^{1}$ and V. Bláha ${ }^{3}$ \\ ${ }^{1}$ 2nd Department of Internal Medicine, Hematology, Medical Faculty and Teaching Hospital, Charles University, \\ Sokolská 408, 50005 Hradec Králové, Czech Republic \\ ${ }^{2}$ Department of Analytical Chemistry, Faculty of Pharmacy, Charles University, Heyrovského 1203, \\ 50005 Hradec Králové, Czech Republic \\ ${ }^{3}$ Department of Metabolic Care and Gerontology, Faculty of Medicine and Teaching Hospital in Hradec Králové, \\ Charles University, Sokolská 481, 50005 Hradec Králové, Czech Republic
}

Correspondence should be addressed to M. Bláha, blaham@email.cz

Received 26 July 2010; Revised 19 September 2010; Accepted 13 October 2010

Academic Editor: Leonid Medved

Copyright ( $) 2011$ M. Bláha et al. This is an open access article distributed under the Creative Commons Attribution License, which permits unrestricted use, distribution, and reproduction in any medium, provided the original work is properly cited.

Background. Using our statin analysis method, it was possible to uncover a significant drop in statin levels (atorvastatin, simvastatin, and metabolites) after extracorporeal LDL-cholesterol elimination (EE) in severe familial hypercholesterolemia (FH). The purpose of this work was to identify the mechanism underlying this drop and its clinical significance as well as to propose measures to optimize a pharmacotherapeutical regimen that can prevent the loss of statins. Methods. Ultra High Performance Liquid Chromatography (UHPLC) connected to the triple quadrupole MS/MS system was used. Patients. A group of long-term treated patients (3-12 years of treatment) with severe FH (12 patients) and treated regularly by LDL-apheresis (immunoadsorption) or haemorheopheresis (cascade filtration) were included in this study. Results. After EE, the level of statins and their metabolites decreased (atorvastatin before/after LDL-apheresis: 8.83/3.46 nmol/l; before/after haemorheopheresis: $37.02 / 18.94 \mathrm{nmol} / 1$ ). A specific loss was found (concentration of atorvastatin for LDL-apheresis/haemorheopheresis: 0.28/3.04 nmol/l in washing fluids; $11.07 \mathrm{nmol} / \mathrm{l}$ in filters). To prevent substantial loss of statin concentrations, a pharmacotherapeutic regimen with a longer time interval between the dose of statins and EE is recommended (15 hours). Conclusions. A specific loss of statins was found in adsorbent columns and filters. The decrease can be prevented by the suggested dosage scheme.

\section{Introduction}

Familial hypercholesterolemia (FH) is a severe metabolic disorder that leads to accelerated atheromatosis [1]. A complete change in lifestyle, a strict diet, and intensive combined pharmacotherapy are necessary in patients with $\mathrm{FH}$ and provide very good results in most patients [2]. In spite of these measures to resolve $\mathrm{FH}$, extracorporeal elimination (EE) of low-density lipoprotein cholesterol (LDL-cholesterol) is still necessary for patients who are homozygous for the disease-causing gene and a small number of heterozygous patients. EE is carried out using currently accepted methods, including heparin-induced extracorporeal LDL precipitation
(HELP), direct adsorption of lipoprotein (DALI), LDLapheresis (immunoadsorption), dextran-sulfate adsorption (lipopheresis), and hemorheopheresis (cascade filtration) [3-6]. Statins, combined with other cholesterol-lowering agents (ezetimibe, fibrates, or bile acid sequestrants), are the most efficient and the most widely used pharmacotherapeutics for FH treatment [7-9]. The therapeutic range of statins is relatively low (typically $10-80 \mathrm{mg} /$ day) $[10,11]$, and patients with severe $\mathrm{FH}$ must take the maximally tolerable dose of statins. Such doses have a potent cholesterollowering effect and significantly reduce the morbidity and mortality associated with coronary heart disease, as shown by many clinical trials $[9,12-14]$. However, some statins 
exhibit a number of adverse effects, such as myopathy or rhabdomyolysis [1, 15-18]. Nevertheless, therapeutic drug monitoring is not routinely carried out for patients treated with statins. Patients are only advised to report the development of muscle aches, pains, or weakness, which increases the risk of clinically important adverse effects. The determination of statin levels in biological fluids is clinically important, but measurements are technically difficult and quite expensive; therefore, statin levels cannot be routinely determined in a common biochemical laboratory. Such determination requires specialized laboratory processing and analytical equipment; many important and interesting data were described elsewhere [19-22].

A sensitive and selective analytical method for statin analysis during EE was developed in our laboratories. After the initial analyses, we observed considerable decreases in statin levels after EE in some patients. This decrease could adversely influence our patients, although no data were found in the literature to allow us to evaluate the possible effects of this decrease. The aims of the present work were to evaluate the quantity of statin loss (as well as active metabolites of statins) during EE, to identify the mechanism of statin loss during EE (possibly captured also in absorbers or filters), and to propose measures to reduce losses, such as adjustments to the pharmacotherapeutical regimen.

\section{Materials and Methods}

2.1. Chemicals and Reagents. Working standards of simvastatin were obtained from Sigma Aldrich (Prague, Czech Republic). Working standards of simvastatin acid, atorvastatin lactone and atorvastatin, p-hydroxyatorvastatin, o-hydroxyatorvastatin, deuterium-labeled simvastatin (D6methyl groups), and deuterium-labeled atorvastatin (D5phenyl ring) were purchased from Toronto Research Chemicals (Ontario, Canada).

Acetic acid (reagent grade), ammonium (reagent grade), and acetonitrile (LC-MS grade) were purchased from Sigma Aldrich. High-performance liquid chromatography (HPLC) grade water was obtained with a Milli-Q reverse osmosis Millipore (Bedford, MA, USA) and met the requirements of the European Pharmacopoeia.

2.2. Therapeutic Procedures. All patients treated in the long term with EE in the Czech Republic were chosen for the purpose of this study. The following two EE approaches were used in twelve patients.

2.2.1. LDL-Apheresis. The patients were treated by LDLapheresis based on the principle of immunoadsorption. The procedure consisted of the following two main steps: (1) separation of plasma using a Cobe-Spectra continual centrifugation separator (Cobe, Denver, USA) followed by (2) passage of plasma through a pair of Lipopak 400 adsorbers (Pocard, Moscow, Russia) with sheep antibodies against apolipoprotein $\mathrm{B}$. The adsorbers are placed into an automatic adsorption-desorption device (Adasorb, Medicap, Ulrichstein, SRN). The pair of columns was alternated until the cholesterol level was significantly below the limit. The target LDL-cholesterol value used at the start of this research was less than $1 \mathrm{mmol} / \mathrm{L}$, but the procedure has become much more effective, with a target value decreased to below $0.5 \mathrm{mmol} / \mathrm{L}$ (normal values typically range from 1.5 to $3.36 \mathrm{mmol} / \mathrm{L}$ ).

2.2.2. Hemorheopheresis (Cascade Filtration). Cell-free plasma was obtained by high-speed centrifugation using a Cobe-Spectra separator, as described above. The plasma was then pumped through the second level (filters). Based on their properties, Evaflux $4 \mathrm{~A}$ and $5 \mathrm{~A}$ (Kuraray) filters were used in our modification of cascade filtration (named "hemorheopheresis" in our paper). The filters contained hollow fibers made from ethylene-vinyl alcohol material with a pore size of 0.03 or $0.04 \mu \mathrm{m}$. Anticoagulation was performed with ACD-A (Baxter, Munich, Germany) and an initial intravenous bolus injection of $4000 \mathrm{IU}$ of heparin. The body plasma volume was calculated using the Cobe-Spectra separator computer, and we washed 1.5X of the body plasma volume.

2.2.3. Monitored Parameters. To monitor the safety, efficiency, and cost-effectiveness of the therapy, the patients were regularly tested for a set of basic hematological, biochemical, and immunological parameters (data not shown) [23-26].

This study was approved by the institutional ethics committee, and all examinations were in agreement with the principles of the currently valid version of the Helsinki declaration. All patients signed an informed consent form.

2.3. Chromatography and Mass Spectrometry. The previously developed and validated ultra-high-performance liquid chromatography tandem mass spectrometry (UHPLCMS/MS) technique was applied to measure the levels of simvastatin, atorvastatin, and their metabolites in serum and washing liquids [27]. A UHPLC system (Waters, Prague, Czech Republic) was used for the purposes of this study, and the system consisted of an ACQ-binary solvent manager and an ACQ-sample manager. An MS/MS triple quadrupole system was also used for the purposes of this study. A Quattro Micro apparatus (Micromass, Manchester, GB) was equipped with a multimode ionization source (ESCI). Following SRM (selected reaction monitoring) transitions were monitored: atorvastatin $(559>440$ and $559>466)$, and atorvastatin D5 (564 > 445), p-hydroxyatorvastatin (575 > 440 and $575>466)$, o-hydroxyatorvastatin $(575>440$ and $575>466)$, atorvastatin lactone (541 > 448 and $541>422$ ).

2.4. Preparation of Standard Solutions and Samples. The standard stock solutions used to measure statin levels were prepared according to the procedure described in [27]. Stock solutions were further diluted by the mobile phase (for stability reasons, the $\mathrm{pH}$ of the solution was maintained between 4.0 and 5.0 to prevent interconversion). Serum samples were prepared using solid phase extraction (SPE) with a Discovery DSC-18 SPE support (SPE procedure I). An internal standard $(100 \mu \mathrm{L})$ was added to $900 \mu \mathrm{L}$ of the serum 
samples containing the analytes. The mixture was loaded onto a Discovery DSC-18 sorbent previously activated with $1 \mathrm{~mL}$ of acetonitrile and conditioned with $1 \mathrm{~mL}$ of $0.1 \mathrm{M}$ ammonium acetate buffer, $\mathrm{pH}$ 4.5. An SPE cartridge with a loaded sample was washed two times with $1 \mathrm{~mL}$ of $0.1 \mathrm{M}$ ammonium acetate, $\mathrm{pH} 4.5$, then once with $1 \mathrm{~mL}$ of a mixture of acetonitrile $[0.01 \mathrm{M}$ ammonium acetate buffer, $\mathrm{pH} 4.5(15: 85, \mathrm{v} / \mathrm{v})]$. Finally, the analytes were eluted with $1 \mathrm{~mL}$ of acetonitrile [ $0.1 \mathrm{M}$ ammonium acetate buffer, $\mathrm{pH} 4.5$ $(95: 5, \mathrm{v} / \mathrm{v})]$. The eluate was filtered through a $0.20 \mu \mathrm{m}$ PTFE filter, and the sample was injected onto the UHPLC system [27].

The samples of washing liquids were prepared using a modified version of SPE procedure I. Four cycles were usually used during LDL-apheresis. First, the plasma of the patient was administered through the adsorbent columns to bind cholesterol. Subsequently, in the washing step, cholesterol was washed away by glycine acidification. In this phase, statins potentially retained in the adsorbent columns might be released as well. Therefore, the glycine washing solution was also analyzed by UHPLC-MS/MS. The sample was obtained during the first cycle, when the highest amount of cholesterol was present in the plasma. During the hemorheopheresis procedure, all washing liquid was collected in the waste bag and examined by UHPLCMS/MS. A homogenization step was necessary to obtain representative samples of washing liquids. Therefore, waste bags were shaken for 30 minutes. Prior to the extraction of washing liquids, the samples were centrifuged at $3000 \times \mathrm{g}$ for 10 minutes. If necessary, the samples were also filtered through a $0.20 \mu \mathrm{m}$ PTFE filter. For SPE, $5 \mathrm{~mL}$ of supernatant was used. Conditioning, washing, and elution steps were performed according to SPE procedure I.

Preparation of samples from filters was performed as follows. After the filtering procedure, all liquids were removed from the filters. The filters were then washed in acetonitrile to remove any remaining statins. All liquid was homogenized by shaking. Subsequently, the samples were centrifuged at $3000 \times \mathrm{g}$ for 10 minutes prior to extraction of the washed filters. If necessary, the samples were also filtered through $0.20 \mu \mathrm{m}$ PTFE filters. For SPE, $5 \mathrm{~mL}$ of supernatant was used. Conditioning, washing, and elution steps were performed according to SPE procedure I. However, it was not possible to conduct this experiment quantitatively because some amount of liquid always remained in the filters, so the measurements were only an approximate value.

2.5. Patients. The patient pool included all long-term treated patients undergoing EE procedures in the Czech Republic. This set of 12 patients was gradually generated after 1996 . The patients were treated for 3-12 years (average: $7.2 \pm$ 2.96 years, range: $2-11.5$, median: 7.25). The group of 12 patients consisted of 7 men and 5 women. The average age of the patients was $47 \pm 16.6$ years (range: $21-63$, median: 52 years). The clinical phenotype of $\mathrm{FH}$ was characterized by an increased level of total plasmatic cholesterol and LDLcholesterol as well as by the occurrence of xanthomas and premature symptoms of ischemic heart disease. The MedPed criteria [28], using thresholds for total cholesterol and LDL cholesterol levels above the 95th percentile specific to the Czech population [29], and the individual's age and family history were applied. Furthermore, DNA-based evidence of a mutation in the low-density lipoprotein receptor (LDLR) gene was used as the criterion for homozygous FH. None of the patients carried a mutation in the apolipoprotein $\mathrm{B}$ (APOB) gene.

All patients were treated with high-dose statins $(40 \mathrm{mg}$ simvastatin or $40-80 \mathrm{mg}$ atorvastatin daily). One patient was treated with combined fenofibrate ( $200 \mathrm{mg}$ daily) and a statin, two patients were treated with combined biliary acidbinding resins ( $6 \mathrm{~g}$ daily) and a statin, and 10 patients were treated with combined Ezetrol (ezetimibe, $10 \mathrm{mg}$ daily) and a statin. Rosuvastatin was not available in the Czech Republic during the study period.

EE procedures were performed regularly, with three homozygous FH patients receiving EE every 10-14 days and other hypercholesterolemic patients receiving EE every 3-4 weeks. Five patients presented an increased level of lipoprotein (a) $(>0.30 \mathrm{nmol} / \mathrm{L})$, with an average level of $1.20 \pm$ $0.84 \mathrm{nmol} / \mathrm{L}$ (range: $0.40-2.42 \mathrm{nmol} / \mathrm{L}$, median: $1.1 \mathrm{nmol} / \mathrm{L}$ ). The average body mass index was $26.99 \pm 6.2$ (range: 18.7-42.6, median: 26.35). Nine patients suffered from hypercholesterolemia of Fredrickson phenotype IIa. These patients were either genetically confirmed as homozygous patients with a defective LDLR gene or heterozygous $\mathrm{FH}$ patients. Three patients displayed abnormally high levels of cholesterol and triglycerides. Two patients had a phenotype consistent with Fredrickson IIb. One patient, who was simultaneously treated for type II diabetes mellitus, had a phenotype consistent with Fredrickson IIb-IV.

2.6. Statistical Evaluation. The significance of differences before and after extracorporeal therapy was examined by the Mann-Whitney $U$ test, using the NCSS 2004 statistical software (Number Cruncher Statistical Systems, Kaysville, USA). Differences were considered significant for $P \leq 0.05$.

\section{Results}

Lipoprotein levels were relatively stable in all patients at the time of examination. The final measurements showed the average total cholesterol to be $7.49 \pm 1.61 \mathrm{nmol} / \mathrm{L}$, LDLcholesterol to be $5.2 \pm 1.39 \mathrm{nmol} / \mathrm{L}$, and HDL-cholesterol to be $1.56 \pm 0.43 \mathrm{nmol} / \mathrm{L}$.

3.1. Preliminary Experiments. First, preliminary experiments were performed to verify the applicability of the UHPLCMS/MS method in determining the influence of EE procedures on statin concentrations in biological fluids. In total, 36 samples were analyzed (before and after the EE procedure), including three samples taken from each of twelve patients at regular intervals. Eleven patients were treated with atorvastatin and one with simvastatin during the preliminary study. From the preliminary results, it was evident that intraindividual variability among the particular analyses was low. Therefore, the method was found to be convenient and reliable. The UHPLC-MS/MS method validation was 
performed according to the International Conference on Harmonization (ICH) guidelines for validation [29].

3.2. Detailed Study. Plasma statin concentrations were measured regularly over the course of the study. In total, 170 samples (85 pairs, collected twice from each patient during 2009) were analyzed in all patients when they attended the procedures. For each patient, samples were always analyzed in pairs for concentrations of statins and their metabolites, with one sample taken before the procedure and the other sample taken after the procedure. Only eleven of the twelve patients were admitted to this study because one patient, who was treated with simvastatin, had previously switched to rosuvastatin therapy when it became available in the Czech Republic. In the following study, only patients treated by atorvastatin were observed.

The results from the study are shown in Table 1. After the EE procedures, clinically significant reductions of statins and their metabolites were observed. The concentration of atorvastatin in the serum decreased by an average of $47.2 \%$, p-OH-atorvastatin by $50.0 \%$, o-OH-atorvastatin by $37.9 \%$, and atorvastatin lactone by $49.1 \%$. The changes in concentrations of statins and their metabolites were evaluated statistically using the Wilcoxon nonparametric $t$ test. The difference in mean concentration before and after the procedures was significant for the patients as a group (see Table 1). However, the two EE procedures differ; LDLapheresis is an APOB-specific treatment, and hemorheopheresis is a nonspecific filtration procedure. Therefore, it was necessary to evaluate the results separately. Upon individual evaluation of each EE procedure, we found that the statin and metabolite levels differed significantly before and after LDL-apheresis (Table 1). Results obtained for the hemorheopheresis group were also significant, except those for one metabolite (p-OH-atorvastatin). During LDLapheresis, the atorvastatin serum concentration decreased by $60.8 \%, \mathrm{pOH}$-atorvastatin by $59.0 \%, \mathrm{o}-\mathrm{OH}$-atorvastatin by $44.5 \%$, and atorvastatin lactone by $58.9 \%$. During hemorheopheresis, the atorvastatin serum concentration decreased by $48.8 \%$, p-OH-atorvastatin by $43.60 \%$, o-OHatorvastatin by $44.0 \%$, and atorvastatin lactone by $41.6 \%$.

3.3. The Mechanism of the Statin Drop. Considering the relatively large decrease of serum statin concentrations in all patients studied, further experiments were performed to test the hypothesis that adsorbent columns retain statins during LDL-apheresis and that filters retain statins during hemorheopheresis. To test this hypothesis, 25 samples were collected and subjected to UHPLC-MS/MS analysis. In addition, 22 samples of waste liquids were examined to evaluate each patient twice. The results of this experiment are presented in Table 2. The average concentrations of statins in washing liquids were substantially lower than the serum levels (the atorvastatin concentration during LDLapheresis was $0.28 \pm 0.20 \mathrm{nmol} / \mathrm{L}$, during hemorheopheresis was $3.04 \pm 3.58 \mathrm{nmol} / \mathrm{L}$, and for all patients was $1.34 \pm$ $2.51 \mathrm{nmol} / \mathrm{L}$ ). An analysis of LDL-apheresis was performed on the total amount of waste liquid; however, the waste liquid was only obtained from the first cycle (about $900 \mathrm{~mL}$ ), during which the cholesterol retained in the adsorbent column was washed out with glycine. As stated above, both adsorbent columns worked together as a pair, and each column was typically filled and washed out four times. In total, $3600 \mathrm{~mL}$ of glycine was used, so measurements from all cycles were not technically possible. However, statins are considered to be present in the highest amounts in washing liquids during the first cycle. In contrast, during hemorheopheresis, it was possible to analyze all of the washing liquid $(500-1500 \mathrm{~mL}$ of liquid contained in a bag). The volume of washing liquid during hemorheopheresis varied, depending on the number of times the Evaflux filter was washed, which was controlled by pressure feed-back. If the pressure increased above safe limits, the filter would wash back into the waste bag. The number of washing cycles depended mainly on the fibrinogen level of the patient.

The measurements for the filter matrix (three samples) revealed substantial retention of statins as well. From the matrix, the median atorvastatin level in the eluate was $7.83 \mathrm{nmol} / \mathrm{L}$, the median $\mathrm{p}-\mathrm{OH}$-atorvastatin level was $1.20 \mathrm{nmol} / \mathrm{L}$, the median $\mathrm{o}-\mathrm{OH}$-atorvastatin level was $7.71 \mathrm{nmol} / \mathrm{L}$, and the median atorvastatin-lactone level was $3.83 \mathrm{nmol} / \mathrm{L}$ (Table 3 ). The measurements for the eluate from the filter matrix were performed in triplicate only, due to technical difficulties and economic demands.

The correlation between the changes of statin levels (atorvastatin, $\mathrm{p}-\mathrm{OH}$-atorvastatin, $\mathrm{o}-\mathrm{OH}$ atorvastatin, and atorvastatin lactone) and changes of both the total cholesterol levels and LDL-cholesterol levels in the group of patients taking the statins $2-4$ or 15 hours before the procedures was tested using Pearson's coefficient, and the same analysis was used to examine relative correlation (software STATISTICA $8)$. No significant correlation was found $(P>0.05)$.

\section{Discussion}

Advances in biotechnology bring new knowledge and new methods that can be used in many branches of medicine [3034]. One such advance is extracorporeal therapy, which can be either non-specific (such as plasma exchange) or specific (such as immunoadsorption). This type of procedure can constitute an important and sometimes decisive component of FH therapy. EE with regard to medical, technical, and economic demands is indicated in cases when classical methods fail.

Our study revealed a significant decrease in atorvastatin and simvastatin levels after EE using LDL-apheresis and hemorheopheresis. The decrease was observed for the parent compound as well as for the metabolites (except for p$\mathrm{OH}$-atorvastatin in the hemorheopheresis group) and the interconversion products. After EE, the concentrations were about half of the initial concentrations. The question of clinical importance of statin level fluctuation after EE is very interesting. The notion that $\mathrm{EE}$ reduces plasma levels of statins is, at face value, an important issue. However, in reality the low bioavailability of statins and the fact that their LDL-lowering efficacy is not correlated with area under the curve concentrations from 0 to 24 hours after dosing [35] 


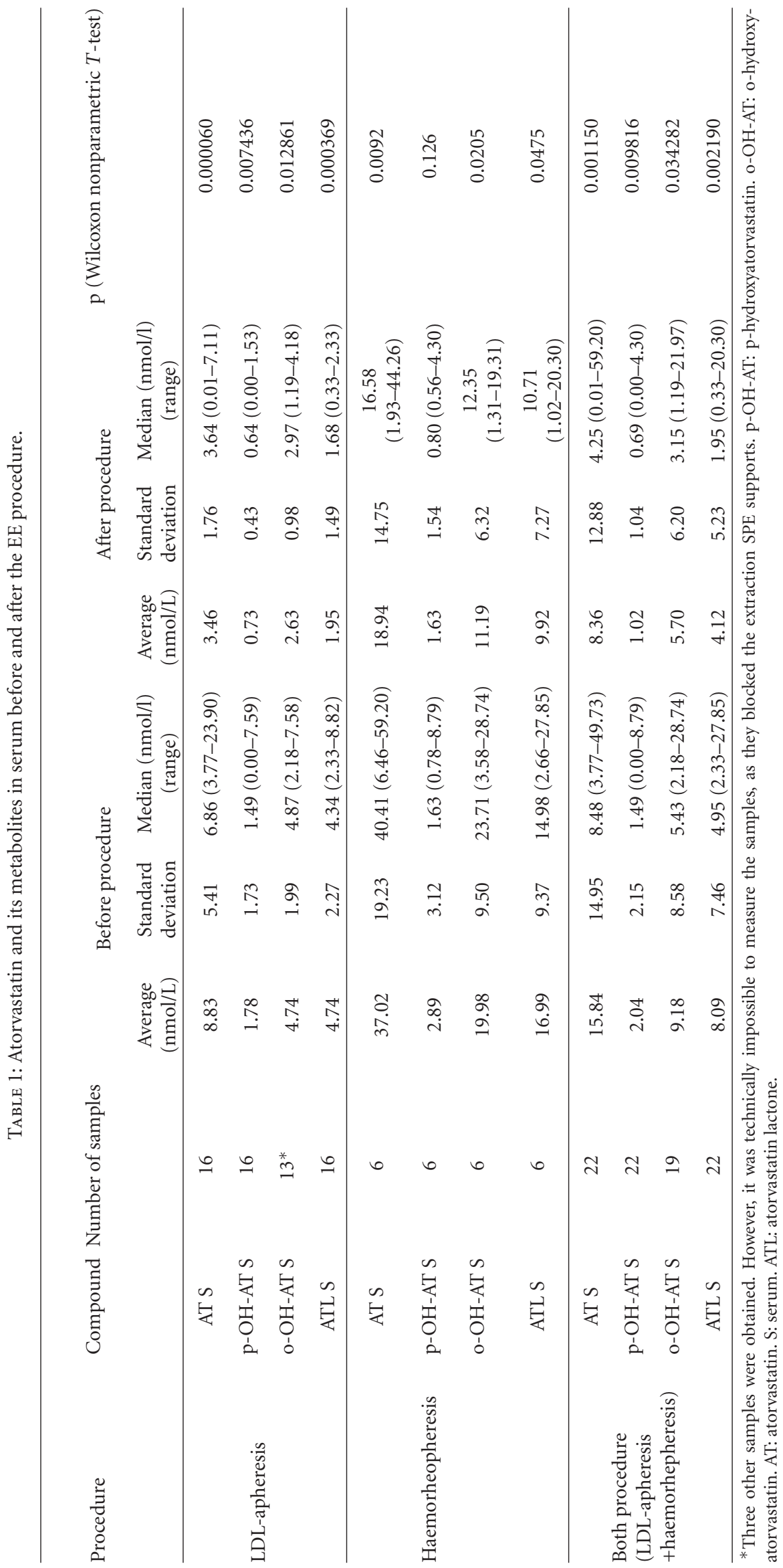


TABLE 2: Atorvastatin and its metabolites in waste fluids.

\begin{tabular}{|c|c|c|c|c|c|}
\hline $\begin{array}{l}\text { Samples from the } \\
\text { procedure }\end{array}$ & Compound & Number of samples & Average (nmol/L) & Standard deviation & $\operatorname{Median}(\mathrm{nmol} / \mathrm{L})$ (range) \\
\hline \multirow{4}{*}{ LDL-apheresis } & AT & 15 & 0.28 & 0.20 & $0.22(0.00-0.77)$ \\
\hline & p-OH-AT & 15 & 0.07 & 0.07 & $0.06(0.00-0.25)$ \\
\hline & o-OH-AT & 15 & 0.09 & 0.11 & $0.06(0.00-0.39)$ \\
\hline & ATL & 15 & 0.15 & 0.18 & $0.12(0.00-0.74)$ \\
\hline \multirow{4}{*}{ Hemorheopheresis } & AT & 7 & 3.04 & 3.58 & $2.57(0.21-12.05)$ \\
\hline & p-OH-AT & 7 & 0.37 & 0.37 & $0.29(0.00-1.06)$ \\
\hline & o-OH-AT & 7 & 1.72 & 1.33 & $1.38(0.05-4.11)$ \\
\hline & ATL & 7 & 2.52 & 1.85 & $2.23(0.96-6.38)$ \\
\hline \multirow{4}{*}{$\begin{array}{l}\text { Both procedures } \\
\text { (Hemorheopheresis } \\
\text { +LDL-apheresis) }\end{array}$} & $\mathrm{AT}$ & 22 & 1.34 & 2.51 & $0.34(0.00-12.05)$ \\
\hline & p-OH-AT & 22 & 0.19 & 0.27 & $0.08(0.00-1.06)$ \\
\hline & o-OH-AT & 22 & 0.71 & 1.13 & $0.12(0.00-4.11)$ \\
\hline & ATL & 22 & 0.91 & 1.51 & $0.18(0.00-6.38)$ \\
\hline
\end{tabular}

p-OH-AT: p-hydroxyatorvastatin, o-OH-AT: o-hydroxyatorvastatin, AT: atorvastatin, ATL: atorvastatin lactone.

TABLE 3: Atorvastatin and its metabolites in the matrix filter.

\begin{tabular}{lcccc}
\hline Compound & Number of samples & Average $(\mathrm{nmol} / \mathrm{L})$ & Standard deviation $(\mathrm{SD})$ & Median $(\mathrm{nmol} / \mathrm{L})(\mathrm{range})$ \\
\hline AT & 3 & 11.07 & 12.32 & $7.83(0.70-24.69)$ \\
p-OH-AT & 3 & 1.18 & 0.98 & $1.20(0.19-2.15)$ \\
o-OH-AT & 3 & 8.28 & 8.25 & $7.71(0.33-16.80)$ \\
ATL & 3 & 6.32 & 7.51 & $3.83(0.38-14.76)$ \\
\hline
\end{tabular}

p-OH-AT: p-hydroxyatorvastatin, o-OH-AT: o-hydroxyatorvastatin, AT: atorvastatin, ATL: atorvastatin lactone.

suggest that reducing plasma levels of statins by apheresis would not adversely affect plasma LDL levels. Also in our study the correlation was not found between statin levels and their LDL-lowering effect. But other data indicate that high plasma levels of statins are associated with myotoxicity, which implies that lowering statin levels with lipoprotein apheresis might be useful in that context. There are also data indicating that the effect of statins is not only hypolipidemic, but pleiotropic [36]. Statin dose/level lowering is in relation to the many unwanted pathophysiological mechanisms, such as in coronary heart disease complications [37], beginning of arrhythmias [38] statins can also influence some renal disorders [39] and play an important role in endothelial or enzymatic cascade activities inclusive of pathological chain "inflammation - thrombocyte activation-thrombogenesis" [40]. It can be concluded that maximum tolerated doses of statins and corresponding high and stable statin levels are desirable in patients with severe $\mathrm{FH}$.

Statins and their metabolites, which may also be effective in lowering cholesterol levels (e.g., atorvastatin hydroxymetabolites), bind to blood proteins, and several of these statin carriers can also be eliminated during an EE procedure. The levels of statins in biological fluids are very low because statins have a high first-pass metabolism. Following dosing with conventional statins widely used in therapy (atorvastatin, simvastatin, and lovastatin), only about 5\% of the dosed statin reaches the systemic circulation. Typical plasma concentrations of statins are $\mathrm{ng} / \mathrm{mL}$ levels. The concentrations of therapeutically effective metabolites of atorvastatin are even lower, typically $0.1-20 \mathrm{ng} / \mathrm{mL}[41,42]$.

The differences in the statin decrease between LDLapheresis and hemorheopheresis are likely related to the different treatment approaches and the duration of the procedures. Similarly, the concentrations of statins before the procedure were higher in the hemorheopheresis group. Patients treated by hemorheopheresis took atorvastatin in the morning, followed by EE within 2-4 hours. Atorvastatin is quickly absorbed after oral administration, and a maximum plasmatic concentration is reached within 1-2 hours [41]. Therefore, the samples from these patients were taken shortly after the maximum plasmatic concentration of statins was reached. Patients treated by LDL-apheresis took the statin dose the evening before $\mathrm{EE}$, so the samples were obtained approximately 15 hours after dosing, which is longer than the mean elimination half-life of 14 hours $[41,42]$. Only one patient treated by LDL-apheresis took the statin dose in the morning, which, in his daily regimen, meant at 4 A.M. For this patient, LDL-apheresis was performed within 5-6 hours of dosing. Therefore, the atorvastatin concentration before EE was found to be substantially higher in this patient (average of three measurements $=13.65 \mathrm{nmol} / \mathrm{L}$ ) as compared with the average of all patients treated by LDL-apheresis $(8.83 \mathrm{nmol} / \mathrm{L})$. However, the atorvastatin concentration before EE was lower in this patient than in patients treated by hemorheopheresis $(37.02 \mathrm{nmol} / \mathrm{L})$. The time interval between dosage and $\mathrm{EE}$ 
dramatically impacted the measurements of statin concentrations. Similarly, the duration of EE also affected the statin concentrations. The time interval between the two blood collections for the determination of statin concentration before and after EE was 3-4 hours in the hemorheopheresis group and 5-6 hours in the LDL-apheresis group.

The pharmacokinetic properties of statins are well known under standard conditions. Our finding of the importance of time interval between blood withdrawals (before and after EE) on the reduction of statin concentrations could be based on pharmacokinetics, unless the metabolism of statins differs between EE procedures. It would be possible to determine whether the time period is unimportant or decisive or if it is the only factor influencing the decrease of statin concentration. Unfortunately, no information about changes in metabolism during EE was found in the literature. However, it may be considered that the EE procedure is a type of stressful situation, which may change the pharmacokinetic decrease of statin concentrations over time. Therefore, a more precise evaluation of measurements of washing liquids and filters was undertaken in our work. LDL-apheresis is a specific type of EE, in which $\mathrm{APOB}$ becomes caught in adsorbent columns together with compounds bound to APOB. Our results revealed that there was only an average of $0.28 \pm 0.20 \mathrm{nmol} / \mathrm{L}$ of atorvastatin in washing liquids. Taking into account an average decrease of atorvastatin of $5.37 \mathrm{nmol} / \mathrm{L}$ in serum after LDL-apheresis, the retention of statins in washing liquids was less important. It should be noted that washing liquids were examined only for the first cycle, although four cycles are typically performed. The concentration of statins in washing liquids after hemorheopheresis (on average, $3.04 \mathrm{nmol} / \mathrm{L}$ ) was found to be higher than after LDL-apheresis, and the concentration was $11.07 \mathrm{nmol} / \mathrm{L}$ in the matrix of filters. The atorvastatin in the serum decreased by an average of $18.08 \mathrm{nmol} / \mathrm{L}$ following hemorheopheresis. The amount of atorvastatin retained in filters and washing liquids was therefore relatively more important than after LDL-apheresis. The absolute amount of retained statin following hemorheopheresis was higher as compared with LDL-apheresis. This result is in agreement with the nonspecificity of hemorheopheresis, which is actually a filtration procedure. The results can be summarized in the following: even though the period with lower levels of statins is not long (lasting only until the next dose of statin) and although these levels are not clinically dangerous (especially in LDL-apheresis), a certain amount of statins is retained during $\mathrm{EE}$, and it is convenient to take a statin dose in the evening before EE (at least 15 hours before $\mathrm{EE}$ ), especially in the case of hemorheopheresis. EE is then performed after the mean half-life elimination time of statin; thus, the absolute loss of statin is lower. We have shown (see above) that the absolute loss of statin is lower in the patients taking a statin dose at least 15 hours before EE than in patients taking the dose $2-4$ hours before EE. It can be hypothesized that the reason why statin losses decrease with increasing the time interval between statin medication and EE is simple-they are practically cleared in a longer time after the mean life-time. Administration of medication in the morning followed by $\mathrm{EE}$ at the time of maximum statin plasma concentration is not ideal from this point of view. Exceptional cases that require the patient to take the drug in the morning on the day of EE can be resolved by the recommendation to take the drug after EE.

\section{Conclusion}

The UHPLC-MS/MS analytical method developed for the determination of statins present in biological materials appears to be adequately sensitive, precise, and highly selective for the purpose of studying patients with $\mathrm{FH}$ after EE. After EE, the concentrations of simvastatin and atorvastatin, as well as their metabolites and interconversion forms, were decreased. This outcome is conditioned by the decrease in concentration over time (according to pharmacokinetic properties), the type of procedure applied, and, most likely, other factors as well. However, we observed specific levels of statins in the washing liquids and filters. This amount was more important after hemorheopheresis. Therefore, it is convenient to prolong the interval between statin dosage and EE to greater than the half-life of the statins (dose $\sim 6$ P.M. the evening before the procedure). In contrast, a morning dose of statins on the day of EE is inconvenient, especially for hemorheopheresis.

\section{Acknowledgments}

This work was supported by a Grant from the Internal Grant Agency, Ministry of Health, Czech Republic, No. NS/9743-4, MZO 00179906, MSM 0021620820.

\section{References}

[1] J. Thompsen and P. D. Thompson, "A systematic review of LDL apheresis in the treatment of cardiovascular disease," Atherosclerosis, vol. 189, no. 1, pp. 31-38, 2006.

[2] H. Borberg, "Quo vadis haemapheresis: current developments in haemapheresis," Transfusion and Apheresis Science, vol. 34, no. 1, pp. 51-73, 2006.

[3] H. Borberg, "26 Years of LDL-apheresis: a review of experience," Transfusion and Apheresis Science, vol. 41, no. 1, pp. 49-59, 2009.

[4] G. R. Thompson, "LDL apheresis," Atherosclerosis, vol. 167, no. 1, pp. 1-13, 2003.

[5] G. R. Thompson, "Recommendations for the use of LDL apheresis," Atherosclerosis, vol. 198, no. 2, pp. 247-255, 2008.

[6] R. Klingel, T. Fassbender, C. Fassbender, and B. Göhlen, "From membrane differential filtration to lipidfiltration: technological progress in low-density lipoprotein apheresis," Therapeutic Apheresis, vol. 7, no. 3, pp. 350-358, 2003.

[7] J. Wlodarczyk, D. Sullivan, and M. Smith, "Comparison of benefits and risk sof rosuvastatin versus atorvastatin from a meta-analysis of head-tohead randomized controlled trials," American Journal of Cardiology, vol. 102, no. 12, pp. 16541662, 2008.

[8] D.-J. Yang and L. S. Hwang, "Study on the conversion of three natural statins from lactone forms to their corresponding hydroxy acid forms and their determination in Pu-Erh tea," Journal of Chromatography A, vol. 1119, no. 1-2, pp. 277-284, 2006. 
[9] V. Bláha, D. Solichová, Z. Zadák et al., "Imunoadsorption LDL-apheresis in the treatment of hypercholesterolemia: five years of research and clinical experience," Nutrition, vol. 18, p. $211,2002$.

[10] R. L. Lins, K. E. Matthys, G. A. Verpooten et al., "Pharmacokinetics of atorvastatin and its metabolites after single and multiple dosing in hypercholesterolaemic haemodialysis patients," Nephrology Dialysis Transplantation, vol. 18, no. 5, pp. 967-976, 2003.

[11] S. Bellosta, R. Paoletti, and A. Corsini, "Safety of statins: focus on clinical pharmacokinetics and drug interactions," Circulation, vol. 109, no. 23, pp. 50-57, 2004.

[12] Y. Shitara and Y. Sugiyama, "Pharmacokinetic and pharmacodynamic alterations of 3-hydroxy-3-methylglutaryl coenzyme A (HMG-CoA) reductase inhibitors: drug-drug interactions and interindividual differences in transporter and metabolic enzyme functions," Pharmacology and Therapeutics, vol. 112, no. 1, pp. 71-105, 2006.

[13] F. M. Sacks, "The relative role of low-density lipoprotein cholesterol and high-density lipoprotein cholesterol in coronary artery disease: evidence from large-scale statin and fibrate trials," American Journal of Cardiology, vol. 88, no. 12A, pp. 14N-18N, 2001.

[14] S. Bertolini, G. B. Bon, L. M. Campbell et al., "Efficacy and safety of atorvastatin compared to pravastatin in patients with hypercholesterolemia," Atherosclerosis, vol. 130, no. 1-2, pp. 191-197, 1997.

[15] J. M. Horacek, M. Tichy, R. Pudil et al., "Multimarker approach to evaluation of cardiac toxicity during preparative regimen and hematopoietic cell transplantation," Neoplasma, vol. 55, no. 6, pp. 532-537, 2008.

[16] J. M. Horacek, M. Tichy, R. Pudil, and L. Jebavy, "Glycogen phosphorylase $\mathrm{BB}$ could be a new circulating biomarker for detection of anthracycline cardiotoxicity," Annals of Oncology, vol. 19, no. 9, pp. 1656-1657, 2008.

[17] A. Šmahelová, R. Hyšpler, T. Haas, A. Tichá, V. Bláha, and Z. Zadák, "Effect of atorvastatin on non-cholesterol sterols in patients with type 2 diabetes mellitus and cardiovascular disease," Pharmacological Research, vol. 51, no. 1, pp. 31-36, 2005.

[18] T. Nakamura, E. Sato, N. Fujiwara et al., "Co-administration of ezetimibe enhances proteinuria-lowering effects of pitavastatin in chronic kidney disease patients partly via a cholesterolindependent manner," Pharmacological Research, vol. 61, no. 1, pp. 58-61, 2010.

[19] E. Pastorini, R. Rotini, M. Guardigli et al., "Development and validation of a HPLC-ES-MS/MS method for the determination of glucosamine in human synovial fluid," Journal of Pharmaceutical and Biomedical Analysis, vol. 50, no. 5, pp. 1009-1014, 2009.

[20] N. Lindegardh, J. Tarning, P. V. Toi et al., "Quantification of artemisinin in human plasma using liquid chromatography coupled to tandem mass spectrometry," Journal of Pharmaceutical and Biomedical Analysis, vol. 49, no. 3, pp. 768-773, 2009.

[21] W. W. Bullen, R. A. Miller, and R. N. Hayes, "Development and validation of a high-performance liquid chromatography tandem mass spectrometry assay for atorvastatin, ortho-hydroxy atorvastatin, and para-hydroxy atorvastatin in human, dog, and rat plasma," Journal of the American Society for Mass Spectrometry, vol. 10, no. 1, pp. 55-66, 1999.

[22] J.-H. Lee, Y.-A. Woo, I.-C. Hwang et al., "Quantification of CKD-501, lobeglitazone, in rat plasma using a liquidchromatography/tandem mass spectrometry method and its applications to pharmacokinetic studies," Journal of Pharmaceutical and Biomedical Analysis, vol. 50, no. 5, pp. 872-877, 2009.

[23] M. Bláha, M. Cermanová, V. Bláha et al., "Safety and tolerability of long lasting LDL-apheresis in familial hyperlipoproteinemia," Therapeutic Apheresis and Dialysis, vol. 11, no. 1, pp. 9-15, 2007.

[24] M. Bláha, J. Krejsek, C. Andrýs et al., "Adhesive selectin molecules, MCP-1 and endotelin-1 during long-lasting LDL apheresis in familial hyperlipoproteinemia," Therapeutic Apheresis and Dialysis, vol. 9, no. A29, 2005.

[25] M. Bláha, A. Strasova, L. Ungerman et al., "Significance of non-invasive cardiovascular examinations for the evaluation of extracorporeal LDL-cholesterol elimination efficacy," Transfusion Medicine and Hemotherapy, vol. 35, supplement, p. 80, 2008.

[26] M. Bláha, E. Rencová, J. Studnička et al., "Cascade filtration in the therapy of the dry form of age-related macular degeneration," Therapeutic Apheresis and Dialysis, vol. 13, no. 5, pp. 453-454, 2009.

[27] L. Nováková, H. Vlčková, D. Šatínský et al., "Ultra high performance liquid chromatography tandem mass spectrometric detection in clinical analysis of simvastatin and atorvastatin," Journal of Chromatography B, vol. 877, no. 22, pp. 2093-2103, 2009.

[28] R. R. Williams, S. C. Hunt, M. C. Schumacher et al., "Diagnosing heterozygous familial hypercholesterolemia using new practical criteria validated by molecular genetics," American Journal of Cardiology, vol. 72, no. 2, pp. 171-176, 1993.

[29] M. Samánek and Z. Urbanová, "Cholesterol and triglyceride levels and their development from 2 to 17 years of age," Casopís Lékarů Ceských, vol. 136, no. 12, pp. 380-385, 1997.

[30] R. D. Sleator and C. Hill, "Rational design of improved pharmabiotics," Journal of Biomedicine and Biotechnology, vol. 2009, Article ID 275287, 7 pages, 2009.

[31] L.-H. Ye, H. Zhang, L.-Y. Wu et al., "Anti-Hepatitis B Virus $\mathrm{X}$ protein in sera is one of the markers of development of liver cirrhosis and liver cancer mediated by HBV," Journal of Biomedicine and Biotechnology, vol. 2009, Article ID 289068, 6 pages, 2009.

[32] S. Karray-Chouayekh, F. Trifa, A. Khabir et al., "Clinical significance of epigenetic inactivation of hMLH1 and BRCA1 in tunisian patients with invasive breast carcinoma," Journal of Biomedicine and Biotechnology, vol. 2009, Article ID 369129, 7 pages, 2009.

[33] A. M. Iga, J. H. P. Robertson, M. C. Winslet, and A. M. Seifalian, "Clinical potential of quantum dots," Journal of Biomedicine and Biotechnology, vol. 2007, Article ID 76087, 10 pages, 2007.

[34] A. El Andaloussi and C. Bilhou-Nabera, "New complex chromosomal translocation in chronic myeloid leukaemia: $\mathrm{T}(9 ; 18 ; 22)(\mathrm{q} 34 ; \mathrm{p} 11 ; \mathrm{q} 11)$," Journal of Biomedicine and Biotechnology, vol. 2007, Article ID 92385, 3 pages, 2007.

[35] R. H. Stern, B.-B. Yang, N. J. Hounslow, M. Macmahon, R. B. Abel, and S. C. Olson, "Pharmacodynamics and pharmacokinetic-pharmacodynamic relationships of atovarstatin, an HMG-CoA reductase inhibitor," Journal of Clinical Pharmacology, vol. 40, no. 6, pp. 616-623, 2000.

[36] J. Rossi, P. Jonak, L. Rouleau, L. Danielczak, J.-C. Tardif, and R. L. Leask, "Differential response of endothelial cells to Simvastatin when conditioned with steady, non-reversing pulsatile or oscillating shear stress," Annals of Biomedical Engineering. In press. 
[37] C. Heeschen, C. W. Hamm, U. Laufs, S. Snapinn, M. Böhm, and H. D. White, "Withdrawal of statins increases event rates in patients with acute coronary syndromes," Circulation, vol. 105, no. 12, pp. 1446-1452, 2002.

[38] N. Wanahita, J. Chen, S. Bangalore et al., "The effect of statin therapy on ventricular tachyarrhythmias: a meta-analysis," American Journal of Therapeutics. In press.

[39] C. Zoja, D. Corna, E. Gagliardini et al., "Adding a statin to a combination of ACE inhibitor and ARB normalizes proteinuria in experimental diabetes which translates into full renoprotection," American Journal of Physiology. In press.

[40] R. S. Rosenson and C. C. Tangney, "Antiatherothrombotic properties of statins: implications for cardiovascular event reduction," Journal of the American Medical Association, vol. 279, no. 20, pp. 1643-1650, 1998.

[41] Goodman \& Gilman's, The Pharmacological Basis of Therapeutics, McGraw-Hill, New York, NY, USA, 11th edition, 2006.

[42] S. C. Sweetman, Martindale: The Complete Drug Reference, Pharmaceutical Press, London, UK, 35th edition, 2007. 

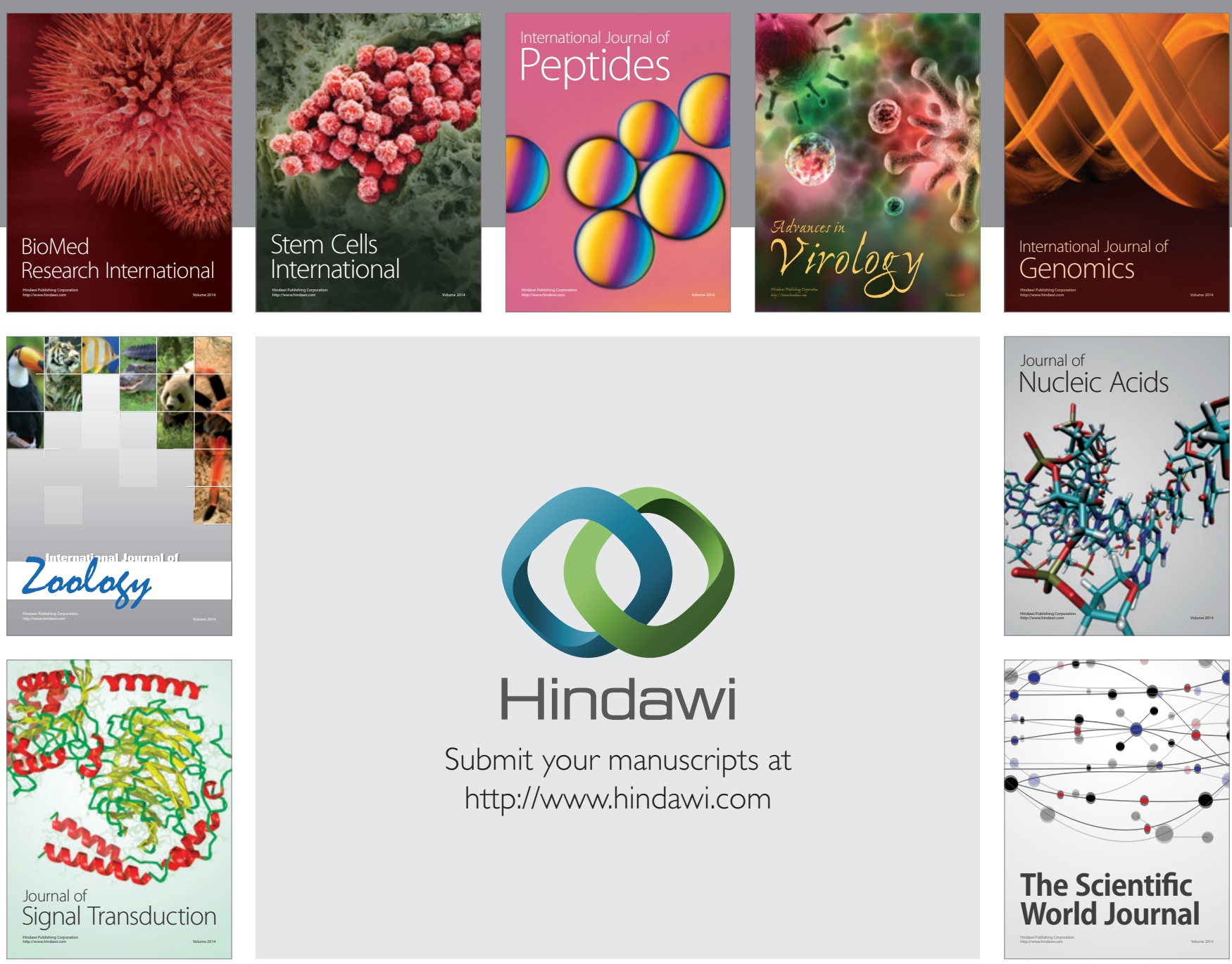

Submit your manuscripts at

http://www.hindawi.com
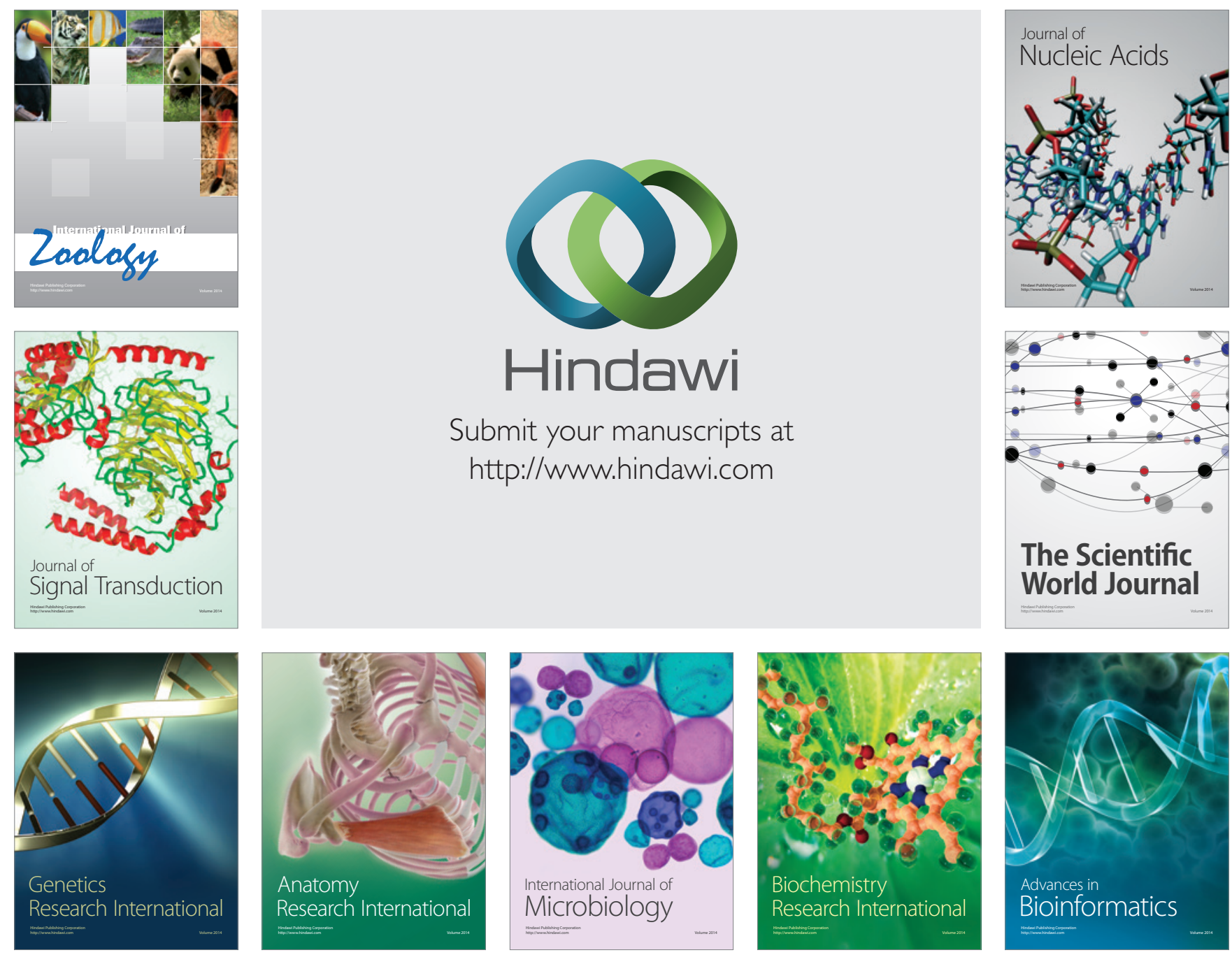

The Scientific World Journal
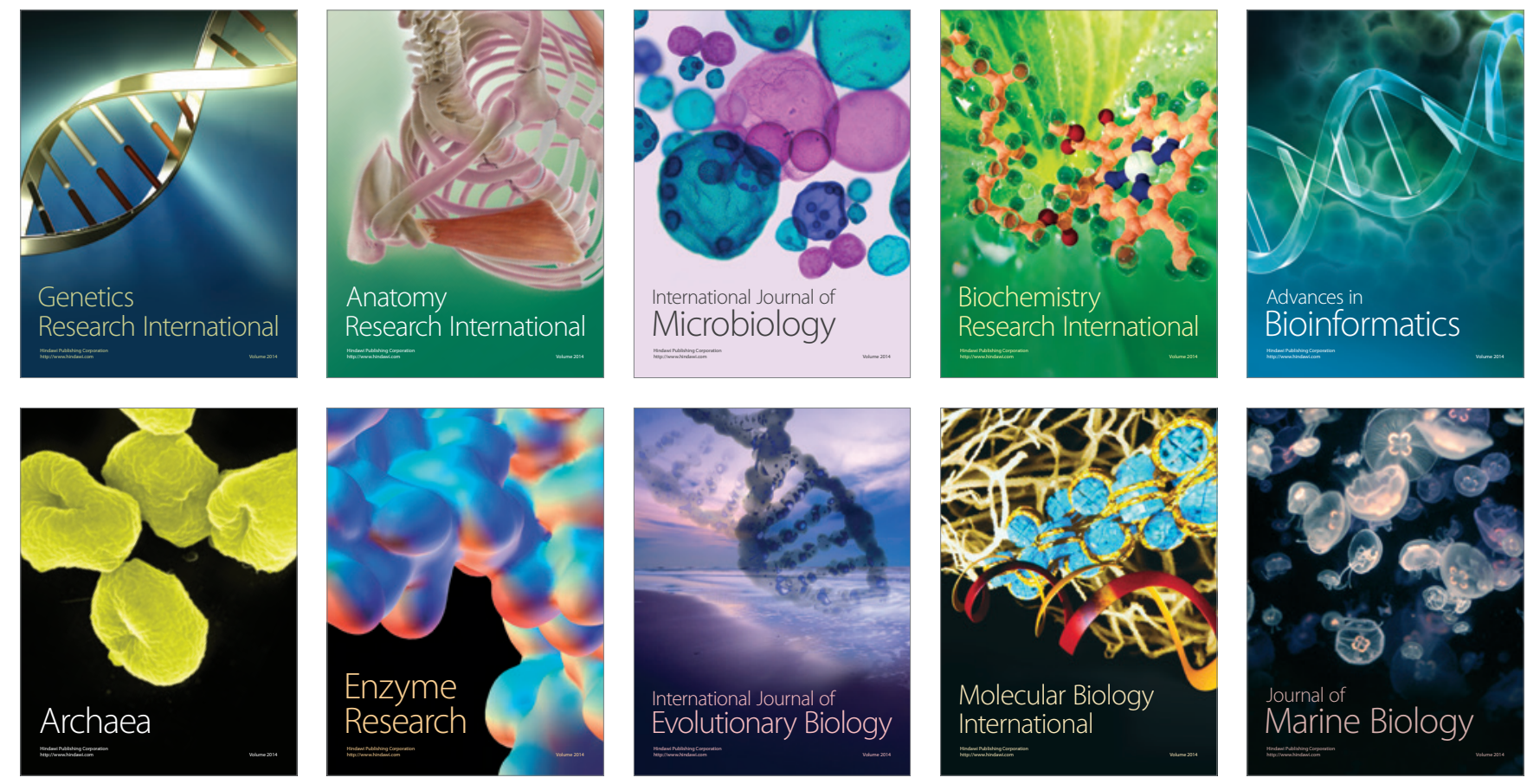